\title{
Managing a Patient with Post-Heart Transplant Associated with COVID-19: A Case Report from Qatar
}

Nissar Shaikh ${ }^{1}$, Mohamad Khatib ${ }^{1}$, Aya Elghazali ${ }^{1}$, Mohammad Alwraidat ${ }^{1}$, Fahmi Othman $^{1}$, Muna AlMaslamani ${ }^{2}$, and Abdulqadir Nashwan ${ }^{1}$

${ }^{1}$ Hamad Medical Corporation

${ }^{2}$ Hamad Medical Corp

July 9, 2021

\begin{abstract}
This is a case of an elderly female with comorbidities and a history of cardiac transplant 13 years back, presented with diarrhea and diagnosed with COVID-19. She was hospitalized and found to have a cardiac injury and urinary tract infection, treated with antibiotics, antivirals, immunosuppressives, and required non-invasive ventilatory support.
\end{abstract}

Managing a Patient with Post-Heart Transplant Associated with COVID-19: A Case Report from Qatar

Short title: Post-Heart Transplant and COVID-19

Nissar Shaikh ${ }^{1}$, Mohamad Y. Khatib ${ }^{2}$, Aya S. Elghazali ${ }^{2}$, Mohammad A. Al Wraidat ${ }^{2}$, Fahmi Othman $^{3}$, Muna A. Almaslamani ${ }^{4}$, Abdulqadir J. Nashwan ${ }^{2}$

1. Surgical Intensive Care Department, Hamad General Hospital (HGH), Hamad Medical Corporation (HMC), Doha, Qatar.

2. Medical Intensive Care Department, Hazm Mebaireek General Hospital (HMGH), Hamad Medical Corporation (HMC), Doha, Qatar.

3. Department of Cardiology and Cardiovascular Surgery, Heart Hospital, Hamad Medical Corporation (HMC), Doha, Qatar.

4. Communicable Diseases Center (CDC), Hamad Medical Corporation (HMC), Doha, Qatar.

Corresponding author:

Mr. Abdulqadir J. Nashwan

anashwan@hamad.qa

Tel: $(+974) 40240487$

Mob: (+974) 66473549

P.O.Box 3050 Doha, Qatar

\section{Abstract}

This is a case of an elderly female with comorbidities and a history of cardiac transplant 13 years back, presented with diarrhea and diagnosed with COVID-19. She was hospitalized and found to have a cardiac injury and urinary tract infection, treated with antibiotics, antivirals, immunosuppressives, and required non-invasive ventilatory support. 
Keywords: COVID-19; comorbidities; heart transplant; immunosuppression; intensive care therapy.

\section{Key Clinical Message:}

Post-heart transplant patients can have COVID-19 infection; the patient's outcome and adjustment in immunosuppression therapy depend upon the severity of the COVID-19 infection.

\section{BACKGROUND}

COVID-19 Pneumonia was initially described in China and quickly became a pandemic with high morbidity and significant social and financial loss. (1) More than 70\% of COVID-19 infections are mild, $20 \%$ require hospitalization, and 5\% need intensive therapy. $(2,3)$ Post-organ transplant patients are at a higher risk of developing COVID-19 infection due to immunosuppression. These patients are also at a higher risk for severe COVID-19 infection and its complications than the general population. (4, 5) Cardiac transplant recipients' are at a higher risk of developing COVID-19 infection due to having multiple comorbidities and receiving immunosuppression therapy. (6)

We are reporting a case of COVID-19 infection in cardiac transplant recipient patients as well as reviewing the available literature.

\section{CASE PRESENTATION}

The patient is a 73 years old female with a history of non-ischemic cardiomyopathy prior to orthotropic heart transplantation in 2007, percutaneous coronary intervention (PCI) to the left anterior descending artery (LAD) in 2014, diabetes mellitus, hypertension, obesity, stroke, gouty arthritis, recurrent urinary tract infection (UTI) on treatment. The immunosuppression regimen consisted of tacrolimus $1 \mathrm{mg}$ twice daily on milrinone $200 \mathrm{mg}$ daily infusion at home.

The patient presented on April 2020 to the hospital because she was having loose, watery stools for one day, vomiting, and oliguria in addition to shortness of breath not associated with cough, sore throat, or runny nose. The clinical examination showed temperature $38.4^{\circ} \mathrm{C}$, heart rate of 109 , blood pressure of $149 / 70$, respiratory rate 20 and oxygen saturation $89 \%$ bilateral lung crepitation on auscultation, abdomen soft, no guarding, non-tender grossly normal extremities, no pitting edema, pale palpebral conjunctiva, anicteric sclera, pale lips, and nailbeds. COVID-19 (PCR) was done, which came positive, and urine culture showed $E$. coli and $K$ pneumonia. Chest X-ray showed pulmonary congestion, blunted left cost phrenic angle, suggesting mild pleural effusion/thickening. Notable laboratory values include: $\mathrm{Hb} 7.8 \mathrm{~g} / \mathrm{dl}(12-15 \mathrm{~g} / \mathrm{dl})$, Urea $9.8 \mathrm{mmol} / \mathrm{L}(2.5-7.8 \mathrm{mmol} / \mathrm{L})$, CRP $103.2 \mathrm{mg} / \mathrm{L}(0.0-5.0 \mathrm{mmol})$, Procalcitonin $0.70 \mathrm{ng} / \mathrm{mL}(0.15$ $\mathrm{ng} / \mathrm{mL})$, Troponin $24 \mathrm{ng} / \mathrm{L}(3-10 \mathrm{ng} / \mathrm{L})$, Pro-BNP $1276 \mathrm{pg} / \mathrm{mL}$.

Hence, she was admitted to the intensive care unit (ICU) as a case of COVID-19 infection and UTI; she was started on amlodipine $10 \mathrm{mg}$, aspirin $75 \mathrm{mg}$ once daily, azithromycin $500 \mathrm{mg}$ for 5 days ertapenem $1 \mathrm{~g}$ infusion for 5 days, hydroxychloroquine for 5 days, furosemide $20 \mathrm{mg}$ for 5 days, prednisolone $5 \mathrm{mg}$ for 5 days, pantoprazole $40 \mathrm{mg}$ for 5 days, oseltamivir $75 \mathrm{mg}$ twice daily day 2 to day 5 . On day 2 She was in distress, requiring continuous positive airway pressure (CPAP) therapy, then she was stable on room air alternating with non-invasive ventilation and never required intubation. Her ICU course of admission was for a total five days without any complications; she was subsequently improved and transferred to the medical floor for one-day observation and discharged the next day.

An echocardiogram was done on 29 April 2020 for follow up it showed a mild precordial effusion, no right pulmonary hypertension, Normal global systolic LV function (EF $61 \%$ ). There were no significant valvular abnormalities.

\section{DISCUSSION}

The rate of COVID-19 infection in cardiac transplant patients is double compared to the general population. (7) According to an Italian study, out of 47 post-heart transplant patients with COVID-19 infection, 37\% required hospitalization, and only 8.5\% required intensive care therapy. (7) Another single-centre, case-series 
form the US described the characteristics, treatment, and outcomes of post-heart transplant patients with COVID-19 infection; 28 patients required hospitalization, majority of these patient presented with respiratory symptoms whereas $48 \%$ of these patients had gastrointestinal symptoms. The case fatality rate in recipients of heart transplant who were infected with COVID-19 infection was around 25\%. (6)

Latif et al described that majority of their patients had multiple comorbidities, mainly hypertension, diabetes mellitus, obesity, and chronic kidney disease. In the majority of their patients post COVID-19 infection were complicated by the cardiac injury (77\%), $20 \%$ of their patients required oxygen supplementation, and 7 patients required intensive care therapy with supportive organ management. Seventy-seven percent of patients required reduction in immunosuppression therapy, along with antibiotics and COVID-19 therapy. None of their patients had graft rejection.(6)

According to Biottio et al, $66 \%$ of their patients had a reduction in immunosuppression therapy along with other COVID-19 therapy. (7)

Both above-mentioned studies $(6,7)$ described the mortality in post-heart transplant patients with COVID19 infection to be around $30 \%$ and mainly due to respiratory and other multiple organ failure.

Our patient also had multiple comorbidities, presented with gastrointestinal symptoms, had cardiac injury, and required support with non-invasive ventilation. As in our patient, COVID-19 infection was mild; it did not require any changes or reduction in their immunosuppression therapy, and the patient was discharged home without any complications.

\section{CONCLUSION}

Post-heart transplant patients can have COVID-19 infection; the patient's outcome and adjustment in immunosuppression therapy depend upon the severity of the COVID-19 infection.

\section{Declarations}

\section{Ethics approval and consent to participate}

The article describes a case report. Therefore, no additional permission from our Ethics Committee was required.

\section{Consent for publication}

The consent for publication was obtained.

\section{Availability of data and material}

All data generated or analyzed during this study are included in this published article.

\section{Competing interests}

The authors declare that they have no competing interests.

\section{Funding}

This study was not funded.

\section{Authors' contributions}

NS, MK, AE, MW, FO, MA, AN: Data Collection, Literature Search, Manuscript Preparation

All authors read and approved the final manuscript

\section{Acknowledgments}

Open Access funding provided by the Qatar National Library.

\section{REFERENCES}


1. WHO. Coronavirus disease ( COVID-19): weekly epidemiological update. 2020.

2. Pereira MR, Mohan S, Cohen DJ, Husain SA, Dube GK, Ratner LE, et al. COVID-19 in solid organ transplant recipients: initial report from the US epicenter. American Journal of Transplantation. 2020;20(7):18008.

3. Khatib MY, Peediyakkal MZ, Elshafei MS, Elzeer HS, Ananthegowda DC, Shahen MA, et al. Comparison of the clinical outcomes of non-invasive ventilation by helmet vs facemask in patients with acute respiratory distress syndrome. Medicine. 2021;100(4).

4. Richardson S, Hirsch JS, Narasimhan M, Crawford JM, McGinn T, Davidson KW, et al. Presenting characteristics, comorbidities, and outcomes among 5700 patients hospitalized with COVID-19 in the New York City area. Jama. 2020;323(20):2052-9.

5. Othman F, Abid AR, Abdulkarim S, Khatib MY, Nashwan AJ, Alkuwari M, et al. Right atrial mass in a patient with COVID-19 pneumonia: A case report. Clinical Case Reports. 2021;9(5):e04220.

6. Latif F, Farr MA, Clerkin KJ, Habal MV, Takeda K, Naka Y, et al. Characteristics and outcomes of recipients of heart transplant with coronavirus disease 2019. JAMA cardiology. 2020;5(10):1165-9.

7. Bottio T, Bagozzi L, Fiocco A, Nadali M, Caraffa R, Bifulco O, et al. COVID-19 in heart transplant recipients: a multicenter analysis of the Northern Italian outbreak. Heart Failure. 2021;9(1):52-61. 